\title{
GHS and other Inequalities *
}

\author{
Joel L. Lebowitz
}

Belfer Graduate School of Science, Yeshiva University, New York, N.Y., USA

Received September 16, 1973

Abstract. We use a transformation due to Percus to give a simple derivation of the Griffiths, Hurst, and Sherman, and some other new inequalities, for Ising ferromagnets with pair interactions. The proof makes use of the Griffiths, Kelly, and Sherman and the Fortuin, Kasteleyn, and Ginibre inequalities.

\section{Introduction}

We consider an Ising spin system with ferromagnetic pair interactions; $\sigma_{i}= \pm 1, i \in \Lambda, i=1, \ldots,|\Lambda|$,

$$
H(\boldsymbol{\sigma})=-1 / 2 \sum_{i \neq j} J_{i j} \sigma_{i} \sigma_{j}-\sum_{i} h_{i} \sigma_{i}, \quad J_{i j} \geqq 0,
$$

where $H$ is the energy of the system and $h_{i}$ are external magnetic fields.

The Ursell, or cluster function, $u_{l}\left(i_{1}, \ldots, i_{l}\right)$ play a central role in statistical mechanics. They are given for spin systems, by the relations [1]

$$
u_{l}\left(i_{1}, \ldots, i_{l}\right)=\frac{\partial^{l}}{\partial h_{i_{1}} \ldots \partial h_{i_{l}}} \ln Z(\Lambda ; \boldsymbol{h}, \boldsymbol{J}), \quad l=1, \ldots,|\Lambda|
$$

where we have written $\boldsymbol{h}$ and $\boldsymbol{J}$ for the collections $\left\{h_{i}\right\},\left\{J_{i j}\right\}$ and

$$
Z(\Lambda ; \boldsymbol{h}, \boldsymbol{J})=\operatorname{Tr}_{\{\sigma\}} \exp [-H(\boldsymbol{\sigma})] .
$$

Thus

$$
\begin{gathered}
u_{1}(i)=\left\langle\sigma_{i}\right\rangle, \quad u_{2}(i, j)=\left\langle\sigma_{i} \sigma_{j}\right\rangle-\left\langle\sigma_{i}\right\rangle\left\langle\sigma_{j}\right\rangle, \\
u_{3}(i, j, k)=\left\langle\sigma_{i} \sigma_{j} \sigma_{k}\right\rangle-\left\langle\sigma_{i}\right\rangle\left\langle\sigma_{j} \sigma_{k}\right\rangle-\left\langle\sigma_{j}\right\rangle\left\langle\sigma_{i} \sigma_{k}\right\rangle \\
\\
-\left\langle\sigma_{k}\right\rangle\left\langle\sigma_{i} \sigma_{j}\right\rangle+2\left\langle\sigma_{i}\right\rangle\left\langle\sigma_{j}\right\rangle\left\langle\sigma_{k}\right\rangle, \ldots
\end{gathered}
$$

where \langle\rangle denotes expectations with respect to the measure $\mu(\sigma)$ $=Z^{-1} \exp [-H]$; (we have set the temperature $\beta^{-1}=1$ ).

The Griffiths, Kelly, and Sherman [2] (GKS) inequalities for this system apply when the $h_{i}$ have the same sign for all $i$; say $h_{i} \geqq 0, \boldsymbol{h} \geqq 0$, (similar results hold by symmetry when $h_{i}<0$, all $i$ ). They state

$$
\left\langle\sigma_{A} \sigma_{B}\right\rangle \geqq\left\langle\sigma_{A}\right\rangle\left\langle\sigma_{B}\right\rangle, \quad \boldsymbol{h} \geqq 0
$$

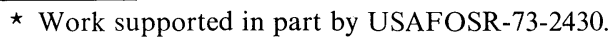


where $A, B \subset \Lambda$ and $\sigma_{A}=\prod_{i \in A} \sigma_{i}$. The more recent Fortuin, Kasteleyn, and Ginibre [3] (FKG) inequalities apply whatever the signs of the $h_{i}$ (assuming always of course that $J_{i j} \geqq 0$ ). FKG consider general increasing functions $f\left(\sigma_{i_{1}}, \ldots, \sigma_{i_{n}}\right), \quad g\left(\sigma_{j_{1}}, \ldots, \sigma_{j_{m}}\right)$, i.e. $f\left(\sigma_{i_{1}}, \ldots, \sigma_{i_{l}}=1, \ldots, \sigma_{i_{n}}\right)$ $\geqq f\left(\sigma_{i_{1}}, \ldots, \sigma_{i_{l}}=-1, \ldots, \sigma_{i_{n}}\right)$. For such functions FKG prove that

$$
\langle f g\rangle \geqq\langle f\rangle\langle g\rangle .
$$

Note that if $g$ is an increasing function $-g$ is a decreasing function and so the inequality in (1.6) is reversed if $f$ is increasing and $g$ is decreasing.

The more general form of the FKG inequalities, which we shall need later, state that if $X$ is a "lattice", e.g. the subsets of $\Lambda$ ordered by inclusion, and if $P(x), x \in X$ is a normalized measure satisfying

$$
P(x) P(y) \leqq P(x \vee y) P(x \wedge y), \quad \text { for all } \quad x, y \in X
$$

then $\langle f g\rangle \geqq\langle f\rangle\langle g\rangle$ whenever $f(x)$ and $g(x)$ are increasing functions, $f(x) \geqq f(y)$ if $x \geqq y$. The expectations are now with respect to the measure $P$.

These inequalities which apply to more general (than pair) ferromagnetic type interactions have found many uses in statistical mechanics [1] (and more recently also in field theory [4]). They can be applied directly to the first two Ursell functions, $u_{1}(i) \geqq 0$, if $\boldsymbol{h} \geqq 0$, by GKS $u_{2}(i, j) \geqq 0$, for arbitrary fields by FKG. For simple proofs of GKS and FKG see [5] and [6] respectively.

There exists also another extremely useful inequality due to Griffiths, Hurst, and Sherman [7] (GHS) which is restricted to ferromagnetic pair interactions,

$$
u_{3}(i, j, k) \leqq 0, \text { for } \boldsymbol{h} \geqq 0 .
$$

(By symmetry $u_{3} \geqq 0$ for $\boldsymbol{h} \leqq 0$.) The derivation of this inequality which is much more specialized than either the GKS or the FKG inequalities, is in many ways also more complicated involving combinatorial analysis, etc. It is the purpose of this note to derive the GHS inequality as part of a whole set of inequalities which are in turn derived, in a fairly simple way from the GKS and FKG inequalities.

\section{Proof of GHS and other Inequalities}

Let $\left\{s_{i}\right\}$ be a "duplicate" set of variables to the $\left\{\sigma_{i}\right\}$ with the same energy as in (1) and let \langle\rangle$^{\prime}$ denote expectations with respect to the measure

$$
\mu(\boldsymbol{\sigma}, s)=Z^{-2}(\Lambda, \boldsymbol{h}, \boldsymbol{J}) \exp [H(\boldsymbol{\sigma})+H(s)] .
$$


Define $q_{i}=\frac{1}{2}\left(\sigma_{i}-s_{i}\right), t_{i}=\frac{1}{2}\left(\sigma_{i}+s_{i}\right), q_{A}=\prod_{i \in A} q_{i}, t_{A}=\prod_{i \in A} t_{i} \cdot q_{i}$ and $t_{i}$ can take on the values, $-1,0,1$ with the constraint that $q_{i}=0 \Rightarrow t_{i}= \pm 1$, $t_{i}=0 \Rightarrow q_{i}= \pm 1$. We then readily find that (dropping the primes on \langle\rangle$^{\prime}$ )

$$
\begin{gathered}
u_{1}(i)=\left\langle t_{i}\right\rangle, \quad u_{2}(i, j)=2\left\langle q_{i} q_{j}\right\rangle \\
u_{3}(i, j, k)=4\left[\left\langle q_{i} q_{j} t_{k}\right\rangle-\left\langle q_{i} q_{j}\right\rangle\left\langle t_{k}\right\rangle\right], \\
u_{4}(i, j, k, l)=8\left[\left\langle q_{i} q_{j} t_{k} t_{l}\right\rangle-\left\langle q_{i} q_{j}\right\rangle\left\langle t_{k} t_{l}\right\rangle\right] \\
-2\left[u_{3}(i, j, k)\left\langle t_{l}\right\rangle+u_{3}(i, j, l)\left\langle t_{k}\right\rangle\right] .
\end{gathered}
$$

Theorem. For Ising system with ferromagnetic pair interactions

$$
\begin{gathered}
\left\langle q_{C}\right\rangle \geqq 0, \quad C \subset \Lambda, \\
\left\langle q_{C} t_{D}\right\rangle \leqq\left\langle q_{C}\right\rangle\left\langle t_{D}\right\rangle, \quad \text { if } \quad \boldsymbol{h} \geqq 0, C, D \subset \Lambda, \\
\left\langle q_{C} q_{D}\right\rangle \geqq\left\langle q_{C}\right\rangle\left\langle q_{D}\right\rangle .
\end{gathered}
$$

Remarks. (i) $\left\langle t_{A}\right\rangle \geqq 0$, and $\left\langle t_{A} t_{\boldsymbol{B}}\right\rangle \geqq\left\langle t_{\boldsymbol{A}}\right\rangle\left\langle t_{\boldsymbol{B}}\right\rangle$, if $\boldsymbol{h} \geqq 0$, is a direct consequence of the GKS inequalities.

(ii) The proof of (2.4) is based on the GKS inequalities alone and is due to Percus [8] who introduced the "duplicate" set of $s$ variables. (2.4) and (2.2) imply $u_{2}(i, j) \geqq 0$ for arbitrary $h_{i}$ without the use of the FKG inequalities [8]. The proof of (2.5) is new and makes use of the GKS and the general form of the FKG inequalities discussed in Section 1. (2.5) together with (2.3) imply the GHS inequality.

Proof. The function $H(\boldsymbol{\sigma})+H(\boldsymbol{s})$ appearing in the measure $\mu(\boldsymbol{\sigma}, \boldsymbol{s})$ in (2.1) becomes in terms of the variables $\boldsymbol{q}$ and $\boldsymbol{t}$,

$$
\begin{aligned}
H_{\Lambda}(\boldsymbol{\sigma})+H_{\Lambda}(\boldsymbol{s}) & =-\left[1 / 2 \sum_{i \neq j} 2 J_{i j} q_{i} q_{j}\right]-\left[1 / 2 \sum_{i \neq j} 2 J_{i j} t_{i} t_{j}+\Sigma 2 h_{i} t_{i}\right] \\
& =\hat{H}_{\Lambda}(\boldsymbol{q} ; 0,2 \boldsymbol{J})+\hat{H}_{\Lambda}(\boldsymbol{t} ; 2 \boldsymbol{h}, 2 \boldsymbol{J})
\end{aligned}
$$

where we have indicated explicitly the dependences of the energy on $\boldsymbol{h}$ and $\boldsymbol{J}$ and use the cap to remind us that $q_{i}$ and $t_{i}$ in (2.6) are not Ising variables since they take the values $-1,0,1$ with the restrictions mentioned earlier. Using (2.6) it is easy to see that for any functions $\phi(\boldsymbol{q})$ and $\Psi(\boldsymbol{t})$

where

$$
\langle\phi(\boldsymbol{q}) \Psi(\boldsymbol{t})\rangle=\sum_{A \subset \boldsymbol{A}} \boldsymbol{P}(A) f(A) q(A),
$$

$$
\begin{aligned}
& P(A)=\left\{\operatorname{Tr}_{q_{I}} \exp \left[-H_{A}(\boldsymbol{q} ; 0,2 \boldsymbol{J})\right]\right\} \\
& \cdot\left\{\operatorname{Tr}_{\boldsymbol{t}_{\boldsymbol{I}}} \exp \left[-H_{\tilde{A}}(\boldsymbol{t} ; 2 \boldsymbol{h}, 2 \boldsymbol{J})\right]\right\} / Z^{2}(\Lambda ; \boldsymbol{h}, \boldsymbol{J}), \\
& f(A)=\left\{\operatorname{Tr}_{\boldsymbol{q}_{\boldsymbol{I}}} \phi(\boldsymbol{q}) \exp \left[-H_{A}(q ; 0,2 \boldsymbol{J})\right]\right\} / Z(A ; 0,2 \boldsymbol{J}), \\
& g(A)=\left\{\operatorname{Tr}_{\boldsymbol{t}_{\boldsymbol{I}}} \Psi(\boldsymbol{t}) \exp \left[-H_{\tilde{A}}(\boldsymbol{t} ; 2 \boldsymbol{h}, 2 \boldsymbol{J})\right]\right\} / Z(\tilde{A} ; 0,2 \boldsymbol{J}) .
\end{aligned}
$$


Here $\tilde{A}$ denotes the complement of $A$ in $\Lambda$ and the subscripts $I$ as well as the absence of a cap on $H_{A}$ and $H_{\tilde{A}}$ indicate that in taking traces in (2.8)-(2.10) over the $q_{i}, i \in A$, and $t_{i}, i \in \tilde{A}$, the $q_{i}$ and $t_{i}$ are to be treated as Ising variables taking on the values \pm 1 only, while $q_{i}=0$ for $i \in \tilde{A}$, $t_{i}=0$ for $i \in A$. Clearly $P(A)$ is the probability with respect to the measure $\mu(\sigma, s)$ that $q_{i}= \pm 1, t_{i}=0$ for $i \in A$, while $f(A)$ is the expectations value of $\phi(q)$ in an Ising system in $A$ with energy $H_{A}(q ; 0,2 J)$. Similarly $g(A)$ is the expectation value of $\Psi(t)$ in an Ising system in $\tilde{A}$ with energy $H_{\tilde{A}}(\boldsymbol{t} ; 2 \boldsymbol{h}, 2 \boldsymbol{J})$.

It is now readily seen that (2.4) holds since in this case $f(A) \geqq 0$ by GKS and $g(A)=1$. To prove (2.5) we note first that it follows from the GKS inequalities that $\left\langle\sigma_{B}\right\rangle, B \subset \Lambda$ is non decreasing as $\Lambda$ is increased, i.e. $\left\langle\sigma_{B}\right\rangle_{\Lambda^{\prime}} \geqq\left\langle\sigma_{B}\right\rangle_{A}$ for $\Lambda^{\prime} \supset \Lambda$ (and $\boldsymbol{h} \geqq 0$ ). Hence for $\phi(\boldsymbol{q})=q_{C}, \Psi(\boldsymbol{t})=t_{D}$, $f(A)$ is an increasing and $g(A)$ a decreasing function of the "size" of $A$, i.e. $f\left(A^{\prime}\right) \geqq f(A), g\left(A^{\prime}\right) \leqq g(A)$ if $A^{\prime} \supset A$. Similarly

where

$$
\left\langle q_{C} q_{B}\right\rangle=\sum_{A \subset A} P(A) \chi(A) \geqq \sum_{A \subset A} P(A) \chi_{1}(A) \chi_{2}(A)
$$

$$
\chi(A)=\operatorname{Tr}_{\boldsymbol{q}_{\mathrm{I}}}\left\{q_{C} q_{D} \exp \left[-H_{A}(\boldsymbol{q} ; 0,2 \boldsymbol{J})\right]\right\} / Z(A ; 0,2 \boldsymbol{J}),
$$

and $\chi_{1}(A), \chi_{2}(A)$ are the corresponding Ising expectations of $q_{C}$ and $q_{B}$ respectively. The inequality in (2.11) follows from GKS which also shows that both $\chi_{1}(A)$ and $\chi_{2}(A)$ are increasing functions on the sets $A$.

Thus (2.5) will be a consequence of the FKG inequalities if we can show that the measure $P$ satisfies (1.7), i.e.

$$
P(A) P(B) \leqq P(A \cup B) P(A \cap B) .
$$

Using (2.8) we see that (2.13) is a consequence of the following lemma.

Lemma. For an Ising spin system with general ferromagnetic interactions,

$$
\begin{gathered}
H_{\Lambda}=-\sum_{Q \subset \Lambda} J_{Q} \sigma_{Q}, J_{Q} \geqq 0 \\
Z\left(\Lambda_{1}\right) Z\left(\Lambda_{2}\right) \leqq Z\left(\Lambda_{1} \cup \Lambda_{2}\right) Z\left(\Lambda_{1} \cap \Lambda_{2}\right)
\end{gathered}
$$

where $Z(\Lambda)=\operatorname{Tr}_{\sigma .} \exp \left[H_{\Lambda}(\boldsymbol{\sigma})\right]$.

Remark. In applying (2.15) to prove (2.13), $\Lambda_{1}=A, \Lambda_{2}=B$ or $\Lambda_{1}=\tilde{A}$, $\Lambda_{2}=\tilde{B}$.

Proof. Setting $\Lambda_{1} \cap \Lambda_{2}=K_{1}, \Lambda_{1} \backslash K_{1}=K_{2}, \Lambda_{2} \backslash K_{1}=K_{3}$ we want to show that

$$
F \equiv \ln Z\left(K_{1} \cup K_{2} \cup K_{3}\right)-\ln \left[Z\left(K_{1} \cup K_{2}\right) Z\left(K_{1} \cup K_{3}\right) / Z\left(K_{1}\right)\right] \geqq 0 .
$$


Now it follows readily from the GKS inequalities that (2.16) holds when there is no interaction between spins on sites in $K_{1}$ and spins on sites in $K_{3}$. Consider now the change in $F$ when an interaction term of the form $J \sigma_{Q_{1}} \sigma_{Q_{3}}, J>0, Q_{1} \subset K_{1}, Q_{3} \subset K_{3}$ is added to the energy of the system. Taking the derivative of $F$ with respect of $J$ we obtain

$$
\frac{\partial F}{\partial J}=\left\langle\sigma_{Q_{1}} \sigma_{Q_{3}}\right\rangle_{K_{123}}-\left\langle\sigma_{Q_{1}} \sigma_{Q_{3}}\right\rangle_{K_{13}}
$$

where we have used the abbreviations $K_{12}=K_{1} \cup K_{2}$, etc. The right side of (2.17) is $\geqq 0$ by GKS and hence $F \geqq 0$. The lemma and the theorem are thus proven.

Remarks. (i) It follows from (2.3) that $u_{4}(i, j, k, l) \leqq 0$ whenever $\left\langle\sigma_{k}\right\rangle$ and $\left\langle\sigma_{l}\right\rangle$ (or, by symmetry the expectation values of any pair of spins in the set $\{i, j, k, l\}$ ) vanish, e.g. $\boldsymbol{h}=0$. Furthermore using the symmetry of $u_{4}$ and the fact that $\left\langle t_{k} t_{l}\right\rangle=\left\langle q_{k} q_{l}\right\rangle$ when $\boldsymbol{h}=0$, we have in this case

$$
\begin{aligned}
& \left|u_{4}(i, j, k, l)\right| \leqq 2 u_{2}(i, j) u_{2}(k, l), \\
& \left|u_{4}(i, j, k, l)\right| \leqq 2\left[u_{2}(i, j) u_{2}(k, l) u_{2}(i, k) u_{2}(j, l)\right]^{1 / 2}, \\
& \left|u_{4}(i, j, k, l)\right| \leqq 2\left[u_{2}(i, j) u_{2}(k, l) u_{2}(i, k) u_{2}(j, l) u_{2}(i, l) u_{2}(j, k)\right]^{1 / 3} .
\end{aligned}
$$

This may be of some relevance to the question of the decay rates of the different Ursell functions [9].

(ii) For $\boldsymbol{h} \geqq 0,\left\langle q_{A}\right\rangle$ is a decreasing and $\left\langle t_{A}\right\rangle$ is an increasing function of the external fields $\left\{h_{i}\right\}$.

(iii) By combining (2.4), (2.5) and the subsequence remark we find that for $\boldsymbol{h} \geqq 0$,

$$
\begin{aligned}
& \left\langle q_{A} t_{B}\right\rangle\left\langle q_{C}\right\rangle \leqq\left\langle q_{A}\right\rangle\left\langle t_{B}\right\rangle\left\langle q_{C}\right\rangle \leqq\left\langle q_{A} q_{C}\right\rangle\left\langle t_{B}\right\rangle, \\
& \left\langle q_{A} t_{B}\right\rangle\left\langle t_{C}\right\rangle \leqq\left\langle q_{A}\right\rangle\left\langle t_{B}\right\rangle\left\langle t_{C}\right\rangle \leqq\left\langle q_{A}\right\rangle\left\langle t_{B} t_{C}\right\rangle .
\end{aligned}
$$

Acknowledgement. I am greatly indebted to J. Percus for communicating to me his method of using the "duplicate" set of variables $s$ prior to publication. I also want to thank D. Ruelle for some useful comments.

\section{References}

1. See for example, Ruelle, D.: Statistical mechanics. New York: Benjamin 1969. — Lebowitz, J. L.: Commun. math. Phys. 28, 313 (1972)

2. Griffiths, R. B.: J. Math. Phys. 8, 478, 484 (1967). — Kelly, D. G., Sherman, S.: J. Math. Phys. 9, 466 (1968)

3. Fortuin, C.M., Kasteleyn, P.W., Ginibre, J.: Commun. math. Phys. 22, 89 (1971)

4. See for example, Simon, B.: Commun. math. Phys. 31, 127 (1973) 
5. Ginibre, J.: Commun. math. Phys. 16, 310 (1970)

6. Cartier,P.: Seminaire Bourbaki, no. 431, 1972/73

7. Griffiths, R.B., Hurst, C. A., Sherman, S.: J. Math. Phys. 11, 790 (1970)

8. Percus, J.: unpublished

9. Duneau, M., Souillard, B., Iagolnitzer,D.: Commun. math. Phys. 31, 191 (1973) and Analyticity and strong cluster properties for classical gases with finite range interaction (preprint)

Communicated by D. Ruelle

\author{
J. L. Lebowitz \\ Belfer Graduate School of Science \\ Yeshiva University \\ 500 West $185^{\text {th }}$ Street \\ New York, N.Y. 10033, USA
}

